\title{
Efeito do treinamento muscular respiratório em pacientes submetidos à colecistectomia
}

\section{Effect of respiratory muscle training in patients undergoing cholecystectomy}

\author{
Claudiane Pedro Rodrigues ${ }^{1}$; Nair Simone de Toledo Costa ${ }^{2}$; Luiz Antonio Alves ${ }^{3}$; \\ Cristiane Golias Gonçalves ${ }^{4}$
}

\section{Resumo}

As cirurgias abdominais altas, como as colecistectomias, tendem a evoluir no pós operatório com distúrbios ventilatórios restritivos. O objetivo deste trabalho foi avaliar a evolução da força muscular inspiratória após o treinamento da musculatura respiratória, por meio do uso de aparelho com carga linear pressórica em pacientes submetidos à colecistectomia convencional. Foram estudados 10 pacientes do sexo feminino, com indicação de colecistectomia, e elas realizaram avaliação da força muscular inspiratória através da medida da Pressão Inspiratória Máxima (PImáx). Deles, seis foram avaliados no pré e pós-operatório (G1 - grupo 1) e realizaram o Treinamento Muscular Respiratório (TMR) com uso do Theshold ${ }^{\circledR}$ ( 2 vezes ao dia no $1^{\circ}$ e no $2^{\circ}$ pós-operatório utilizando $40 \%$ da PImáx), e quatro pacientes foram acompanhados no pós-operátorio como grupo controle (G2 - grupo 2). Os resultados indicam que, apesar da diminuição da PImáx no pós-operatório, houve recuperação da força muscular inspiratória já no primeiro dia de TRM, de 36,7 $\pm 13,7 \mathrm{cmH}_{2} \mathrm{O}$ para $48,0 \pm 13,7 \mathrm{cmH}_{2} \mathrm{O}$ e este aumento foi estatisticamente significante $(\mathrm{p}<0,05)$, o que não ocorreu no grupo controle. Conclui-se que há uma redução na força muscular respiratória após colecistectomia e que o TMR foi satisfatório na melhora de força muscular respiratória.

Palavras-chaves: Colecistectomia. Treinamento muscular respiratório. Fisioterapia.

\begin{abstract}
The upper abdominal surgeries such as cholecystectomy, tend to evolve postoperatively with restrictive ventilatory disorders. The aim of this study was to evaluate the evolution of inspiratory muscle strength after training the respiratory muscles through the use of linear load device with pressure in patients undergoing conventional cholecystectomy. We studied 10 female patients with indication for cholecystectomy who underwent assessment of inspiratory muscle strength by means of the Maximal Inspiratory Pressure (MIP). Of these, six were evaluated preoperatively and postoperatively (G1 - group 1) and conducted Respiratory Muscle Training (RMT) using the Theshold ${ }^{\circledR}$ (twice a day on the 1st and 2nd postoperative day using $40 \% \mathrm{MIP}$ ) and four patients were observed in the post-surgery as a control group (G2 - group 2). The results indicate that despite the decrease in MIP postoperatively, there was recovery of inspiratory muscle strength on the first day of RMT, from $36.7 \pm 13.7 \mathrm{cmH}_{2} \mathrm{O}$ to $48.0 \pm$ $13.7 \mathrm{cmH}_{2} \mathrm{O}$, and this increase was statistically significant $(\mathrm{p}<0.05)$, which did not occur in the control group. We conclude that there is a reduction in respiratory muscle strength after cholecystectomy and that the RMT was considered satisfactory in the improvement of the respiratory muscle strength.
\end{abstract}

Key words: Cholecystectomy. Respiratory muscle training. Physiotherapy.

\footnotetext{
1 Especialista em Fisioterapia cardiorrespiratória; professora do departamento de Fisioterapia da Faculdade de Apucarana - FAP Email: claufisio@yahoo.com.br.

2 Doutora em Ciências Fisiopatologia Experimental (USP); Fisioterapeuta.

3 Mestre em Medicina e Ciências da Saúde, professor do Departamento de Fisioterapia do Centro Universitário Filadélfia - UniFil, Londrina/PR.

${ }^{4}$ Especialista em Fisioterapia Respiratória ; professora do departamento de Fisioterapia da Faculdade de Apucarana - FAP.
} 


\section{Introdução}

As Cirurgias abdominais altas tendem a evoluir no pós-operatório com distúrbios ventilatórios restritivos, tanto em procedimentos convencionais, como nos laparoscópicos (ERICE et al., 1993). A fisiopatogênese desses distúrbios está relacionada principalmente com a inibição reflexa do nervo frênico, induzindo à disfunções diafragmáticas. Outros fatores, porém, devem ser considerados, dentre eles o principal é a dor (RAMOS et al., 2009).

Segundo Ford (1993); Celli (1998) e Siafakas (1999), a incidência de disfunção diafragmática no pós-operatório de cirurgia abdominal baixa é de dois a cinco por cento, enquanto na cirurgia abdominal alta pode aproximar-se de $20-40 \%$. Acredita-se que a disfunção diafragmática seja secundária à lesão direta do músculo e de estruturas próximas a ele, como vasos e nervos, devido à manipulação cirúrgica nessa região. Outros fatores, como o uso de afastador cirúrgico, o tempo de cirurgia prolongado, a dor, as atelectasias, os abscessos subfrênicos, o derrame pleural e a ascite podem estar envolvidos (PACIA; ALDRICH, 1998; CARVALHO et al., 1999).

Geralmente, na colecistectomia convencional, a intervenção cirúrgica é realizada por meio de incisão subcostal à direita e, em alguns casos, é necessário realizar laparotomia, porém, alguns serviços podem optar por incisão longitudinal médio - lateral. Erice et al. (1993), ao comparar colecistectomia laparoscópica com a colecistectomia convencional, mostram que o trauma cirúrgico na parede abdominal pode aumentar a incidência de distúrbio ventilatório pela diminuição da excursão diafragmática. A disfunção diafragmática reduz a ventilação do lobo inferior pulmonar do lado afetado, e diminui o volume corrente, a capacidade vital e a pressão inspiratória máxima (PImáx). Essas alterações podem perdurar por 48 horas após a cirurgia (SIMONNEU; VIVIEN; SARTENE, 1983; PANSARD et al., 1993; GASTALDI et al., 2008).

O retorno dos volumes pulmonares e força muscular respiratória aos valores pré operatórios ocorre de 05 a 10 dias após a colecistectomia laparoscópica, e 12 a 15 dias após a cirurgia convencional. Em cirurgias de grande porte, com comprometimento de vários grupos musculares, ocorrem alterações funcionais importante, com perda de $27 \%$ do volume corrente, $44 \%$ da capacidade vital no $1^{\circ}$ pós operatório, além da diminuição de $32 \%$ na PImáx e $42 \%$ na pressão expiratória máxima (PEmáx). (LIMA, 2002; GASTALDI, 2009).

O comprometimento da força muscular inspiratória leva a uma diminuição do volume pulmonar inspirado e a uma diminuição de volume, associada ao comprometimento da musculatura expiratória, acarreta a diminuição do fluxo expiratório e o prejuízo do mecanismo de tosse, e isso favorece a retenção de secreções pulmonares. (CELLI, 1998; GASTALDI, 2009).

O objetivo deste trabalho é avaliar a evolução da força muscular inspiratória após o treinamento da musculatura respiratória por meio do uso de aparelho, com carga linear pressórica em pacientes submetidos à colecistectomia convencional.

\section{Material e métodos}

Foram estudados prospectivamente 10 pacientes do sexo feminino, sem limite de idade, internados na enfermaria do Hospital da Providência de Apucarana, com indicação de colecistectomia por colecistite crônica ou aguda. Delas, seis foram avaliados no pré-operatório e pós-operatório (G1 - grupo 1) e realizaram Treinamento Muscular Respiratório (TMR); quatro pacientes foram acompanhados no pós-operátorio como grupo controle (G2 - grupo 2). A idade média do G1 era $40 \pm 17$ anos (limites 24 a 67 anos) e no G2 idade média de $45 \pm 17$ anos (limites 29 a 65 anos),

O estudo foi previamente aprovado pelo Comitê de Ética em Pesquisa com Seres Humanos da Faculdade de Apucarana - FAP, com parecer número 95/2008.

O critério de inclusão dos pacientes submetidos 
à cirurgia de Colecistectomia convencional foi apresentarem valores de PImáx. abaixo de $60 \mathrm{cmH}_{2} \mathrm{O}$ no pós - operatório imediato.

Foram excluídos os pacientes que apresentaram no pós-operatório fortes dores no local da incisão cirúrgica em região abdominal, conforme resposta subjetiva, e não conseguiram realizar inspiração profunda, assim como os que foram submetidos a outras intervenções cirúrgicas no decorrer do estudo, e os que não apresentaram a medida da PImáx abaixo de $60 \mathrm{cmH}_{2} \mathrm{O}$ no pós - operatório.

Os pacientes foram submetidos à avaliação da força muscular respiratória, durante os períodos de pré-operatório (G1) e pós-operatório (G2), por meio da medida da PImáx obtida pelo Manovacuômetro com escala operacional em $\mathrm{cmH}_{2} \mathrm{O},(\mathrm{MV}-150 / 300$ da marca Ger-ar). A medida foi realizada com o paciente semi-sentado no leito com membros inferiores estendidos. Inicialmente, pedia-se para o paciente respirar tranqüilamente (volume corrente) e, após três respirações, o paciente realizava uma expiração forçada máxima (volume residual) e, a seguir, um esforço inspiratório estático máximo durante 3 segundos, com oclusão nasal (CELLI, 1998). Neste momento, obtinha-se a medida da força diafragmática (PImáx). Todas as avaliações foram realizadas pelo mesmo examinador e em cada paciente, foram realizadas três medidas, duas vezes ao dia até a alta do paciente que ocorria no segundo dia de pós-operatório, considerando-se apenas a medida de melhor valor. Especificamente no pósoperatório, a medida era realizada após seis horas, aproximadamente. Os pacientes que apresentavam no pós-operatório uma PImáx menor que $60 \mathrm{cmH}_{2} 0$ eram selecionados para treinamento.

Logo após a medida da força diafragmática, os pacientes realizavam o TMR com o aparelho de carga linear pressórica da marca Threshold, composto por uma válvula unidirecional que possibilita a regulagem da pressão sobre a membrana de abertura inspiratória, obtida por uma mola (resistor spring loaded). A carga inspiratória (carga limite de esforço) era obtida pelo ajuste dessa mola pelo terapeuta, graduando dessa forma a resistência ao fluxo inspiratório. O nível da carga inspiratório era prescrito com parâmetro na PImáx obtida previamente, utilizando como carga de treinamento 40\% desta medida (AZEREDO, 1993; PRYOR; WEBBER, 2002).

O TMR foi realizado com o paciente também na posição semi-sentado, com os membros inferiores estendidos. Este era orientado a colocar o bocal do aparelho na boca, com oclusão nasal, solicitava um esforço inspiratório o qual era mantido por cinco segundos. Cada sessão era composta por três séries de cinco cada uma com um minuto de repouso entre cada série. $O$ treinamento, assim como as avaliações da PImáx, eram realizadas duas vezes ao dia totalizando meia hora por dia de treinamento, durante dois dias de hospitalização, sendo no $1^{\circ}$ e no $2^{\circ}$ pós operatório.

Os dados foram organizados em média e desvio padrão por fases (pré e pós-operatório) e aplicado o teste de Shapiro-Wilks para verificar a normalidade dos dados. A análise dos dados foi realizada por meio do test t-pareado. Fixou-se um nível de significância de $5 \%(\mathrm{p}=0,05)$. Os resultados foram expressos em média \pm desvio padrão.

\section{Resultados}

\section{Grupo 1}

A medida da PImáx no pré-operatório foi de $77,5 \pm 7,6 \mathrm{cmH}_{2} \mathrm{O}$ e no pós-operatório foi de $36,7 \pm$ 13,7 $\mathrm{cmH}_{2} \mathrm{O}$ (Tabela 1). A diferença média entre as medidas da PImáx no pré-operatório e pós-operatório no $\mathrm{G} 1$ foi estatisticamente significante $(40,8 \pm 13,6$ $\left.\mathrm{cmH}_{2} \mathrm{O} ; \mathrm{p}<0,05\right)$. Após o treinamento muscular, a medida PImáx apresentou melhora significativa ao índice de significância de 5\%, quando comparada aos pacientes estudados no pré-operatório. A PImáx no primeiro dia de treinamento foi de 48,0 $\pm 13,7$ $\mathrm{cmH}_{2} \mathrm{O}$ e, no segundo,a medida foi de de $54,0 \pm 15,2$ $\mathrm{cmH}_{2} \mathrm{O}(\mathrm{p}<0,05)$, e um paciente já havia recebido alta. 
Tabela 1. Resultados das avaliações da pressão inspiratória máxima de pacientes do sexo feminino, sem limite de idade, com indicação de colecistectomia por colecistite crônica ou aguda. TMR= treinamento muscular respiratório

\begin{tabular}{llcccc}
\hline & $\mathrm{N}$ & PRÉ-OPERATÓRIO & PÓS-OPERATÓRIO & $1^{\circ}$ DIA TMR & $2^{\circ}$ DIA TMR \\
\hline GI & 6 & $77 \pm 8$ & $37 \pm 14 *$ & $48 \pm 14 \dagger$ & $54 \pm 15 \dagger$ \\
GII(Controle) & 4 & $38 \pm 10$ & $45 \pm 6 \dagger$ & $54 \pm 5 \dagger$ \\
GI + GII & 10 & $37 \pm 11$ & $46 \pm 10 \dagger$ & $53 \pm 12 \dagger$ \\
\hline
\end{tabular}

Legenda: * - diferença estatisticamente significativa $(\mathrm{p} \leq 0,05)$ em relação à "PRÉ-OPERATÓRIO"; $\dagger$ - diferença estatisticamente significativa $(\mathrm{p} \leq 0,05)$ em relação à "PÓS-OPERATÓRIO".

\section{Grupo 2}

A medida de PImáx no pós-operatório foi de 37,5 $\pm 9,6 \mathrm{cmH} 2 \mathrm{O}$. Os pacientes que foram estudados apenas no pós-operatório $(n=4)$ a PImáx no primeiro dia de treinamento foi de 45,0 $\pm 5,8$ $\mathrm{cmH} 2 \mathrm{O}$ e no segundo a medida foi de 53,8 $\pm 4,8$ cmH2O (Tabela 1). O test-t mostrou uma diferença estatisticamente significante ao analisar a primeira medida de PImáx no pós-operatório e a última medida após o TMR. $(-16,3 \pm 7,5, \mathrm{p}=0,02)$.

\section{Resultados dos 10 Pacientes que Realizaram Treinamento}

A medida de PImáx antes do treinamento, no pósoperatório imediato, foi de $37 \pm 11 \mathrm{cmH}_{2} \mathrm{O}$. Após o primeiro treinamento, foi de 45,5 $\pm 9,8 \mathrm{cmH}_{2} \mathrm{O}$ e, no segundo, de 52,8 $\pm 11,6 \mathrm{cmH}_{2} \mathrm{O}$ (Tabela 1). A diferença média entre as medidas da Pimáx, antes do TMR e após o último dia de treinamento, foi estatisticamente significante $\left(-11,5 \pm 24,9 \mathrm{cmH}_{2} 0\right.$; $\mathrm{p}<0,05)$.

\section{Discussão}

No presente estudo, houve diferença significativa entre as medidas da PImáx após a colecistectomia, quando comparados com os valores obtidos na fase de pré-operatório $(\mathrm{p}<0,05)$. Para facilitar a compreensão desse resultado, verifica-se que, em porcentagem, ele corresponderia a uma diminuição de $47,75 \%$ da força muscular diafragmática no primeiro pós-operatório.
Ao analisar os dados dos pacientes separadamente pela idade, os pacientes mais jovens apresentaram uma menor diminuição da força diafragmática e uma melhor resposta ao TMR, com valores da PImáx próximos aos valores obtidos no pré-operatório.

De modo análogo ao verificado neste estudo, os autores como Simonneau, Vivien e Sartene (1983); Ford (1993) e Siafakas (1999) observaram a diminuição da função dos músculos respiratórios, no período do primeiro ao terceiro dia de pósoperatório. Essa diminuição pode persistir por uma semana até alcançar o valor normal, o que sugere a ocorrência de disfunção diafragmática.

A disfunção diafragmática, segundo Siafakas (1999), é responsável por grande parte das complicações pulmonares, entre elas a atelectasia e pneumonias. Segundo o autor, a disfunção pode ocorrer devido a vários fatores: local da incisão cirúrgica por afetar a integridade da musculatura; o tempo do procedimento cirúrgico, durante o qual o paciente permanece em posição supina durante todo o procedimento. Além disso, a anestesia pode gerar um bloqueio neuromuscular, e causar diminuição da contração da musculatura respiratória. Clergue et al. (1984) concluíram que o uso da morfina como anestésico gera depressão ventilatória. Conforme Ford et al. (1983), a disfunção diafragmática pode ocorrer também por uma lesão secundária direta dos músculos abdominais e causar uma falência mecânica e a dor no local da incisão cirúrgica, o que contribui para alterações respiratórias. Ayoub et al. (2001) também descrevem que no curso de 
cirurgias abdominais altas ocorre a diminuição da excursão diafragmática devido à incisão cirúrgica, o que gera a diminuição da amplitude inspiratória, principalmente em colecistectomia convencional.

Dentre as possíveis causas que podem ter alterado o valor da PImáx no pós-operatório neste estudo, acredita-se que a dor no local da incisão foi o grande motivo de dificuldade para a obtenção dos valores da PImáx. O paciente apresentava grande dificuldade na mudança de posição, ou seja, de decúbito dorsal para posição semi-sentado, não conseguia realizar facilmente a inspiração máxima e sustentá-la, e o tamanho da incisão cirúrgica também pode ter colaborado para a queda da PImáx no primeiro pós-operatório devido ao medo e a dor associada a inspiração forçada.

Optou-se por realizar as medidas e o treinamento na posição semi-sentada, por ser esta a posição que permite uma melhor descida diafragmática durante a sua contração e assim representar a maior possibilidade de desenvolver força diafragmática. Em decúbito dorsal horizontal, o peso das vísceras poderia interferir na descida do diafragma e o seu desempenho ser questionável. Os pacientes que apresentaram um PImáx menor $60 \mathrm{cmH}_{2} \mathrm{O}$ na posição semi-sentado eram incluídos TMR, já que a medida demonstra fraqueza muscular diafragmática (ZAGELBAUM; PARÉ, 1983; AZEREDO, 1993).

Os músculos respiratórios, como outros músculos esqueléticos, aumentam sua eficiência após o treinamento. Esse aumento teria reflexos na melhora da força e endurance do diafragma, com melhora da função pulmonar. Ford (1993) demonstra a eficácia da fisioterapia em pacientes com complicações pulmonares, após terem sido submetidos à cirurgias abdominais altas ou baixas. Silva, Guedes e Ribeiro (2003) citam como tratamento de complicações pulmonares pós-operatórias a fisioterapia utilizando manobras de inspirações máximas sustentadas, o que nossos resultados comprovaram com a utilização de TMR com o aparelho de carga linear Threshold.

Os resultados indicam que, apesar da diminuição da medida da PImáx no pós-operatório, houve a recuperação da força muscular diafragmática já no primeiro dia de TRM, de $36,7 \pm 13,7 \mathrm{cmH}_{2} \mathrm{O}$ (valor médio no pré-operatório) para 48,0 $\pm 13,7$ $\mathrm{cmH}_{2} \mathrm{O}$ e esse aumento foi significativo $(\mathrm{p}<0,05)$. Isso corresponde a aproximadamente $59,2 \%$ de aumento, com apenas duas sessões diárias de TMR com Threshold. Quando comparados os valores de pré-operatório com os valores do segundo dia de pós-operatório, ou seja, o último dia de TRM, quando foram realizadas três sessões de TMR com Threshold, a medida da PImáx aumentou para $54 \pm$ $15,2 \mathrm{cmH}_{2} \mathrm{O}$.Verificou-se que houve uma melhora também siginificativa $(\mathrm{p}<0,05)$ da força muscular, o que corresponde a aproximadamente $69,7 \%$. Isso nos leva a concluir que provavelmente se um tempo maior de TMR fosse empregado, isto é, se o tempo de internação fosse maior, melhores resultados poderiam ter sido obtidos.

No grupo dois (G2), que corresponde aos pacientes avaliados no pós-operatório, verificou-se uma melhora de $43 \%$ na força muscular comparando os valores da primeira medida da PImáx no primeiro pós-operatório com a última medida da PImáx no segundo dia de pós-operatório. Não foi possível realizar o estudo de todos os pacientes no pré-operatório, devido às dificuldades de conseguir tempo hábil para a conclusão do estudo.

\section{Conclusão}

Conclui-se que há uma redução na força diafragmática após a colecistectomia convencional. Também se verifica que o TMR, com o uso do aparelho de carga linear Threshold utilizando $40 \%$ da PImáx, foi satisfatório na melhora de força muscular respiratória.

\section{Referências}

AYOUB, J.; COHENDY, R.; PRIOUX, J.; AHMAIDI, S.; BOURGEOIS, J. M..; DAUZAT, M.; RAMONATXO, M.; PRÉFAUT, C. Diaphragm movement before and after cholecystectomy: a sonographic study. Anesthesia 
and Analgesia, Baltimore, v. 92, p. 755-61, Mar. 2001.

AZEREDO, C. A. C. Fisioterapia respiratória moderna. 2. ed. São Paulo: Manole, 1993.

CARVALHO, E. M.; MASSAROLLO, P. C. B.; ISERM, M. R. M.; TOLEDO, N. S.; KAWACAMA, J.; MIES, S.; RAIA, S. Pulmonary evolution in conventional liver transplantation with bypass and the piggback method. Transplantation Proceedings, Orlando, v. 31, p. 10641066, 1999.

CELLI, B. The diaphagm and respiratory muscles. Chest surgery clinics of North América, Philadelphia, v. 8, n. 2, p. 207-224, May. 1998.

CLERGUE, F.; MONTEMBAULT, C.; DESPIERRES, O.; GHESQUIERE, M. D.; HARARI, A.; VIARS, P. Respiratory effects of intrathecal Morphine after upper abdominal surgery. Anesthesiology, Philadelphia, v. 61, p. 677-685, Dec. 1984.

ERICE, F.; FOX, G. S.; SALIB, Y. M.; ROMANO, E.; MEAKINS, J. L.; MAGDER, S. A. Diaphragmatic function before and after laparoscopic cholecystectomy. Anesthesiology, Philadelphia, v. 79, p. 966-75, Nov. 1993.

FORD GT, ROSENAL TW, CLERGUE F, WHITELAW, WA. Respiratory physiology in upper abdominal surgery. Clin Chest Med. 1993; 14 (2): 237-251.

FORD GT, WHITELAW WA, ROSENAL TW, CRUSE PJ, GUENTER CA. Diaphragm function after upper abdominal surgery in humans. Am Rev Respir Dis. 1983;127(4):431-6.

GASTALDI, A. C.; MAGALHAES, C. M. B.; BARAÚNA, M. A.; SILVA, E. M. C.; SOUZA, H. C. D. Benefícios da Cinesioterapia respiratória no pósoperatório de colecistectomia laparoscópica. Revista Brasileira de Fisioterapia, v. 12, n. 2, p. 100-106, mar./ abr. 2008.

GASTALDI, A. C.; FREITAS FILHO, G. A.; PEREIRA, A. P. M.; SILVEIRA, J. M. Quantas medidas de pressões respiratórias são necessárias para se obterem medidas máximas em pacientes com tetraplegia? Colunal Columna, v. 8, p. 1-6, 2009.

LIMA, P. A; CARVALHO, E. M.; ISERN, M. R. M.; MASSAROLO, P. C. B.; MIES, S. Mecânica respiratória e oxigenação no transplante de fígado. Jornal de Pneumologia, v. 28, n. 2, p. 39, out. 2008.

LIMA, P.A. Respiratória e oxigenação no transplante de fígado. Boletim Científico do Centro de Estudos e Pesquisa do Hospital Samaritano. São Paulo, v 1, n. 3,Nov.2002.

PACIA, E. B.; ALDRICH, T. K. Assessment of diaphragm function. Chest surgery clinics of North América, Philadelphia, v. 8, n. 2, p. 225-236, Mar. 1998.

PANSARD, J. L.; MANKIKIAN, B.; BERTRAND, M.; KIEFFER, E.; CLERGUE, F.; VIARS, P. Effects of thoracic extradural block on daphragmatic electrical activity and contractility after upper abdominal surgery. Anesthesiology, Philadelphia, v. 78, n. 1, p. 63-71, Jan. 1993.

PRYOR, J. A.; WEBBER, B. A. Fisioterapia para problemas respiratórios e cardíacos. 2. ed. Rio de Janeiro: Guanabara Koogan, 2002.

RAMOS, G. C.; PEREIRA, E.; NETO, S. G.; OLIVEIRA, E. C. Repercussão respiratória funcional após colecistectomia com incisão subcostal: Efeito analgésico da morfina. Revista do Colégio Brasileiro de Cirurgiões, Rio de Janeiro, v. 36, n. 2, p. 139-143, mar./ abr. 2009

SIAFAKAS NM, MITROUSKA I, BOUROS D, GEORGOPOULOS D. Surgery and the respiratory muscles. Thorax.1999;54:458-65

SILVA, E. F.; GUEDES, R. P.; RIBEIRO, E. C. Estudo das repercussões das cirurgias abdominais sobre os músculos respiratórios. Fisioterapia em movimento, Curitiba, v. 16, n. 1, p. 51-56, jan./mar. 2003.

SIMONNEU, G.; VIVIEN, A.; SARTENE, R. Diaphragm dysfunction induced by upper abdominal surgery: role of postoperative pain. The American review of respiratory disease, Baltimore, v. 128, n. 5, p. 899-893, Nov. 1983.

ZAGElBAUM,G. L.; PARÉ, J. A. P. Manual de tratamento intensivo das doenças respiratórias. Rio de Janeiro: Meds, 1983.

Recebido em: 10 de março de 2009 Aceito em: 20 de dezembro de 2010 